\title{
Caractéristiques des virémies persistantes de bas niveau des patients VIH-1 sous traitement antirétroviral de deuxième ligne au CHU Sourô Sanou de Bobo-Dioulasso (Burkina Faso)
}

\author{
Characteristics of Low-Level Persistent viraemias in HIV-1 Patients Treated with Second-Line \\ ARVs at the Sourô Sanou Teaching Hospital of Bobo-Dioulasso (Burkina Faso)
}

\author{
J. Zoungrana $\cdot$ C.G. Kyelem $\cdot$ K. A. Sondo $\cdot$ K.A. Naibi $\cdot$ A. Hema $\cdot$ N.F. Kabore $\cdot$ A. Poda $\cdot$ A. Bado $\cdot$ I. Yaméogo $\cdot$ \\ H. Séré $\cdot$ A.S. Ouedraogo $\cdot$ S. Tani $\cdot$ Z. Tarnagda
}

Reçu le 5 décembre 2017; accepté le 29 août 2018

(C) Société de pathologie exotique et Lavoisier SAS 2018

Résumé Nous décrivons les caractéristiques des patients infectés par le VIH-1 en deuxième ligne de traitement antirétroviral, avec une virémie persistante de bas niveau. Il s'agissait d'une étude rétrospective à visée descriptive, menée du $1^{\text {er }}$ janvier 2010 au 31 décembre 2016, à partir de la cohorte du service des maladies infectieuses du CHU de Bobo-Dioulasso. Ont été inclus les patients infectés par le VIH-1, en deuxième ligne de traitement ARV stable, ayant une observance $\geq 95 \%$ depuis au moins 12 mois, asymptomatiques, avec une charge virale plasmatique comprise entre 50 et 1000 copies $/ \mathrm{ml}$ sur deux prélèvements consécutifs à au moins 3 mois d'intervalle. Sur 244 patients en deuxième ligne de traitement antirétroviral, 79 répondaient à nos critères d'inclusion. L'âge moyen des patients était de $42 \pm$ 10,2 ans. Les femmes $(35,8$ ans) étaient moins âgées que les hommes $(43,8$ ans $)(p=0,001)$. La plupart des patients

J. Zoungrana $(\bowtie) \cdot$ K.A. Naibi · A. Hema N.F. Kabore

A. Poda · I. Yaméogo · H. Séré

Service des maladies infectieuses

du CHU Sanou Sourô Bobo-Dioulasso, Burkina Faso

e-mail : zojacques@yahoo.fr

C.G. Kyelem

Service de médecine interne

du CHU Sanou Sourô Bobo-Dioulasso, Burkina Faso

K. A. Sondo

Service des maladies infectieuses du CHU Yalgado Ouedraogo de Ouagadougou, Burkina Faso

S. Tani $\cdot$ Z. Tarnagda

Institut de recherche des sciences de la santé de Bobo-Dioulasso,

Burkina Faso

A. Bado · A.S. Ouedraogo

Service de bactériologie virologie

du CHU Sanou Sourô Bobo-Dioulasso, Burkina Faso étaient mariés $(48,1 \%)$, parmis lesquels certains vivaient dans des régimes polygames $(23,5 \%)$. La majorité des patients (38/79) de l'étude avaient un taux de CD4 $\leq 200$ cellules $/ \mathrm{mm}^{3}$. La durée médiane du traitement ARV depuis le début de l'histoire thérapeutique était de 4,8 ans (2,511 ans). La charge virale plasmatique supérieure à $10000 \mathrm{copies} / \mathrm{ml}$ au début du traitement $(\mathrm{p}=0,003)$, et la combinaison TDF+FTC+DRV+RTV $(p=0,001)$ étaient associées à la virémie persistante de bas niveau. La réalisation d'un test génotypique de résistance s'impose pour ces patients afin de mieux adapter le traitement antirétroviral.

Mots clés ARV · Virémie persistante de bas niveau · VIH · Hôpital · Bobo-Dioulasso · Burkina Faso

Abstract We describe the characteristics of patients infected with HIV-1 as second-line antiretroviral therapy, with persisting low-level viremia. This was a descriptive retrospective study, conducted from January 1, 2010 to December 31, 2016, from the Cohort of the Infectious Diseases Department of Bobo-Dioulasso University Hospital. Patients infected with HIV-1, a second line of stable ARV treatment, with $\geq 95 \%$ compliance for at least 12 months, asymptomatic with $\mathrm{CVp}$ between 50 and 1000 copies $/ \mathrm{ml}$ in two consecutive samplings at least 3 months apart. Out of 244 patients in second-line therapy, 79 met our inclusion criteria. The mean age of the patients was $42 \pm 10.2$ years. Women (35.8 years) were younger than men ( 43.8 years) $(\mathrm{p}=0.001)$. Most were married $(48.1 \%), 23.5 \%$ of whom were polygamous. The majority of patients (38/79) in the study had a CD4 count of $<200$ cells $/ \mathrm{mm}^{3}$. The median duration of ARV therapy since the beginning of the therapeutic history has been 4.8 (2.5-11 years). CVp greater than 10,000 copies $/ \mathrm{ml}$ at the start of second-line therapy $(\mathrm{p}=0.003)$ and $\mathrm{TDF}+\mathrm{FTC}+\mathrm{DRV}+$ RTV combination $(\mathrm{p}=0.001)$ were associated with persistent 
low viremia. A genotypic resistance test is needed for these patients in order to better adapt the ARV treatment.

Keywords ARV · Low level viremia · HIV · Hospital · Bobo-Dioulasso · Burkina Faso

\section{Introduction}

Malgré les efforts déployés dans la lutte contre l'infection à $\mathrm{VIH}$, celle-ci demeure un problème majeur de santé publique par sa prévalence qui reste particulièrement élevée en Afrique subsaharienne. Dans ces pays, le traitement antirétroviral (ARV) repose sur une approche de santé publique, visant à faciliter l'accès universel. Cette approche permet la simplification et la standardisation de la décision de mise sous ARV et les schémas thérapeutiques. Ainsi elle définit les schémas thérapeutiques de première intention (ou traitement de première ligne) et ceux de deuxième intention (ou traitement de deuxième ligne). Toutefois les patients sous traitement ARV de deuxième ligne sont plus exposés au risque d'échec thérapeutique que ceux sous ARV de première ligne [16]. Les critères de changement de traitement en Afrique sont majoritairement fondés sur la clinique, la numération des lymphocytes $\mathrm{CD} 4$ et parfois la charge virale plasmatique (CVp) et non sur les résultats d'un test génotypique, conduisant au risque d'émergence de virus résistants et parfois l'apparition des mutations croisées sous traitement de deuxième ligne. L'accès à la troisième ligne demeure limité dans les pays en voie de développement $[16,17]$.

Les conséquences d'une réplication virale résiduelle avec une $\mathrm{CVp}$ inférieure à 50 copies $/ \mathrm{ml}$ ne sont pas clairement établies. Cependant la virémie persistante de bas niveau (VPN) comprise entre 50 et 1000 copies $/ \mathrm{ml}$ sous pression de sélection thérapeutique expose au risque de sélection et d'accumulation de mutations de résistance et à une détérioration immunologique pouvant conduire à une progression clinique et à une augmentation du risque de transmission du VIH $[12,19]$.

Au service des maladies infectieuses (SMI) du CHU de Bobo-Dioulasso, 244 patients étaient sous traitement ARV de deuxième ligne en fin 2016. La CVp y est réalisée en routine depuis 2008 . Une proportion non négligeable de patients VIH-1, ayant une observance optimale sous traitement $\mathrm{ARV}$ de deuxième ligne, présente des virémies détectables de bas niveau, entre 50 et 1000 copies $/ \mathrm{ml}$, confirmées sur plusieurs prélèvements consécutifs. Aucune étude n'a cependant été spécifiquement consacrée aux VPN liées au VIH-1 à Bobo-Dioulasso.

Notre étude a donc pour but de décrire les caractéristiques de la VPN chez les patients infectés par le VIH-1, en deuxième ligne de traitement ARV.

\section{Patients et méthodes}

Il s'agissait d'une étude de cohorte rétrospective, descriptive, menée du $1^{\text {er }}$ janvier 2010 au 31 décembre 2016. Ont été inclus les patients adultes infectés par le VIH-1, en deuxième ligne de traitement ARV stable, ayant une observance estimée $\geq 95 \%$ depuis au moins 12 mois, asymptomatiques, avec une CVp comprise entre 50 et 1000 copies/ml sur deux prélèvements consécutifs à au moins 3 mois d'intervalle et suivis au SMI du Centre hospitalier universitaire Sanou Sourô de Bobo-Dioulasso. Les patients infectés par le VIH-2, le VHB et co-infectés VIH-1 /VIH-2, ont été exclus.

Le logiciel ESOPE (évaluation et suivi opérationnel des programmes d'ESTHER), utilisé en temps réel pour le suivi médical des patients au sein du service, a permis le recueil des données sociodémographiques, cliniques, biologiques et thérapeutiques. Ces données ont été recueillies à l'initiation $\mathrm{du}$ traitement de deuxième ligne, puis tous les six mois. Lorsque la $\mathrm{CVp}$ était supérieure à 50 copies $/ \mathrm{ml}$ chez un patient, il était suivi tous les trois mois pour la mesure de l'observance et le contrôle de la CVp.

Le protocole de deuxième ligne du traitement ARV comportait 2 inhibiteurs nucléosidiques de la reverse transcriptase (INRT) non utilisés lors de la première ligne, associés à un inhibiteur de la protéase hautement actif, à savoir le lopinavir/ritonavir ou le darunavir/ritonavir, selon le programme national de lutte contre le VIH/SIDA au Burkina Faso [7,9].

La mesure de l'observance a été faite par un infirmier qui utilisait la méthode du comptage des comprimés. Pour ce faire, les patients ont été priés de rapporter le restant de leur dernier approvisionnement ARV tous les deux mois (délai d'approvisionnement). Les comprimés non rapportés sont supposés avoir été pris par le patient. Le taux d'observance au traitement a été calculé par le rapport entre le nombre de comprimés supposés pris par le patient (prises absorbées) et le nombre de comprimés à prendre (prises à absorber) dans un intervalle de deux mois. Les patients ayant pris au moins $95 \%$ de leur traitement ont été jugés observants. Ces informations sur le niveau d'observance des patients ont été saisies sur une base de données conçue à cet effet sur ACCESS.

La technique Abbott Real Time-PCR avec un seuil de 40 copies $/ \mathrm{ml}$ a été utilisée pour la quantification de la $\mathrm{CVp}$. Les résultats des $\mathrm{CVp}$ étaient saisis sur ESOPE par les médecins au cours des consultations de suivi. Les données extraites à partir du logiciel ESOPE et dans la base de données de la consultation d'observance ont été analysées à l'aide du logiciel Epi Info dans sa version 7.0. Les variables quantitatives ont été présentées par leur moyenne et leur écart type et les variables qualitatives par leur proportion (\%). Les proportions ont été comparées par le test de chi2 de Pearson ou celui exact de Fisher lorsque celui-ci était plus approprié. Le test de Student a été utilisé pour tester l'égalité 
de deux moyennes d'échantillons indépendants et lorsque les conditions d'application n'étaient pas satisfaites, le test non paramétrique de Mann-Whitney était utilisé. Une valeur de $\mathrm{p} \leq 0,05$ a été retenue comme seuil de significativité.

\section{Aspects éthiques}

Avant l'enrôlement dans la file active du service des maladies infectieuses du CHUSS, les patients étaient informés de la saisie de leurs informations médicales dans la base de données et de leur utilisation possible pour des études scientifiques. Chaque patient a donné son consentement.

\section{Résultats}

$\mathrm{Au}$ cours de la période de l'étude, 244 patients ont été mis sous deuxième ligne de traitement $\mathrm{ARV}$ dans le service, dont 79 patients répondaient à nos critères d'inclusion. Le tableau 1 présente les caractéristiques cliniques, biologiques et thérapeutiques des patients en VPN.

Durant cette période, 191 patients avaient une charge virale supérieure à 50 copies $/ \mathrm{ml}$. Parmi eux, 79 patients étaient en VPN, soit une fréquence de 79/244 (32,4\%)

\section{Caractéristiques des patients en VPN}

L'âge moyen des patients était de $42 \pm 10,2$ ans. Les âges extrêmes étaient de 18 et 67 ans. La tranche d'âge des 35 à 45 ans était la plus représentée (36,9\%), suivie de celle de 25 à 35 ans $(32,7 \%$ des cas). Les femmes étaient prédominantes $(62 \%)$ avec un sex-ratio $\mathrm{H} / \mathrm{F}$ de 0,6 . Les femmes étaient en moyenne (35,8 ans) moins âgées que les hommes ( 43,8 ans) $(\mathrm{p}=0,001)$. La population d'étude était constituée de personnes sans emploi (33,7\%), suivies de commerçants, $(29,3 \%)$. La grande majorité des patients résidaient dans la ville de Bobo-Dioulasso (90\%). La plupart étaient mariés (48,1\%), dont (23,5\%) étaient polygames.

Dans notre population d'étude, la suspicion clinique était la circonstance de dépistage la plus fréquente, dans une proportion de $(50,6 \%)$, suivie du dépistage volontaire $(44,3 \%)$.

L'indice de masse corporelle (IMC) moyen des patients indétectables $\left(19,8 \mathrm{~kg} / \mathrm{m}^{2} \pm 5,2 \mathrm{~kg} / \mathrm{m}^{2}\right)$ était plus élevé que celui des patients VPN $\left(17,1 \mathrm{~kg} / \mathrm{m}^{2} \pm 3,3 \mathrm{~kg} / \mathrm{m}^{2}\right)(\mathrm{p}=0,002)$.

La moyenne du taux des lymphocytes TCD4 à l'initiation de la deuxième ligne était de 200 cellules $/ \mathrm{mm}^{3} \pm$ 80,6 cellules $/ \mathrm{mm}^{3}$. La majorité des patients $(38 / 79)$ avait un taux de $\mathrm{CD} 4 \leq 200$ cellules $/ \mathrm{mm}^{3}$. La durée médiane connue du traitement ARV depuis le début de l'histoire thérapeutique était de 4,8 ans (2,5-11 ans). Plus de la moitié des patients (43/79) avait une CVp comprise entre 50 et 200 copies/ml (figure 1$)$. Treize $(21,7 \%$ ) patients avaient une CVp entre 10000 et 100000 copies $/ \mathrm{ml}$ au début du traitement de deuxième ligne. Ces patients avaient un risque moins élevé de VPN que les 35 patients (44\%) ayant une $\mathrm{CVp}$ supérieure à $100000 \mathrm{copies} / \mathrm{ml}(\mathrm{p}=0,003)$. La VPN était plus fréquente chez les patients sous le protocole ténofovir + entrictabine + darunavir + ritonavir que dans les autres protocoles $(p=0,001)$.

\section{Discussion}

Depuis l'avènement des trithérapies, les patients infectés par le VIH présentent majoritairement des $\mathrm{CVp}$ « indétectables ». L'origine des VPN a été largement débattue au sein de la communauté scientifique. Dans ce travail, nous décrivons les caractéristiques de la VPN du VIH-1 chez les patients en deuxième ligne de traitement $\mathrm{ARV}$ au SMI du CHU de BoboDioulasso. La limite de notre étude est la définition retenue pour la caractérisation de la VPN, de même que le nombre de patients inclus. L'étude de Vandenhende réalisée en 2015 en France, portait sur 17902 patients sous traitement ARV; la fréquence de VPN y était de $6,2 \%$; inclus sur la base d'une CVp comprise entre 50 et 500 copies $/ \mathrm{ml}$ [14]. Cependant, nos critères d'inclusion étaient identiques à l'étude de Widera [18] qui retrouvait une VPN chez 20 patients suivis durant 24 mois en Allemagne. Aussi Navarro et al [10] en Espagne sur une cohorte de 300 patients suivis pendant 4,2 ans, avait des critères d'inclusion superposables aux nôtres. La fréquence de la VPN $(32,4 \%)$ dans notre étude était supérieure à celle retrouvée par Navarro et al [6], par Doyle et al [2] et García-Gascó et al [4] qui trouvaient respectivement $17,3 \%, 25 \%$ et $20 \%$ de VPN dans leurs séries. Ces différences pourraient s'expliquer par la taille des échantillons et la durée des études, qui étaient différentes d'une série à l'autre.

La relation entre la durée de l'exposition aux ARV et la suppression virologique est en effet bien connue. Au Burkina Faso, le traitement ARV est standardisé. Cette standardisation est un facteur clé de l'élargissement de l'accès au traitement ARV. Le protocole ARV de deuxième ligne le plus prescrit dans notre étude était le ténofovir + entricitabine + lopinavir/ritonavir $(40 \%)$, suivi de celui associant l'abacavir + didanosine + lopinavir/ritonavir $(32 \%)$. La VPN était fortement associée à la combinaison contenant le darunavir/ritonavir ; plusieurs auteurs en ont fait le constat $[3,11,15]$.

Dans les pays développés il est recommandé de modifier le traitement ARV si la CVp > 500 copies $/ \mathrm{ml}$, du fait d'une accumulation de mutations de résistance aux ARV. En Afrique subsaharienne, particulièrement au Burkina Faso, où l'accès au test génotypique, et autres tests de résistance aux ARV, est limité, la mise en évidence d'une VPN, n'implique donc pas inéluctablement une modification thérapeutique [10]. 
Tableau 1 Répartition des patients selon les caractéristiques cliniques, biologiques et thérapeutiques sous traitement antirétroviral de deuxième ligne / Distribution of patients by clinical, biological, and therapeutic characteristics on second-line antiretroviral therapy

\begin{tabular}{|c|c|c|c|}
\hline Parametres & $\begin{array}{l}\text { CVP }<50 \\
(n=165)\end{array}$ & $\begin{array}{l}50 \geq C V p<1000 \\
(n=79)\end{array}$ & p value \\
\hline \multicolumn{4}{|c|}{ Caractéristiques sociodémographiques } \\
\hline \multicolumn{4}{|l|}{ Sexe : } \\
\hline masculin & $36(0,6)$ & $30(0,4)$ & \multirow[t]{2}{*}{0,008} \\
\hline féminin & $129(0,7)$ & $49(0,3)$ & \\
\hline Age (ans) & $36 \pm 9,5$ & $42,9 \pm 10,2$ & 0,001 \\
\hline \multicolumn{4}{|l|}{ Motif de dépistage : } \\
\hline PTME & $12(75,0)$ & $4(25,0)$ & \multirow{3}{*}{0,9} \\
\hline volontaire & $54(60,7)$ & $35(39,3)$ & \\
\hline suspicion clinique & $99(71,2)$ & $40(28,8$ & \\
\hline \multicolumn{4}{|c|}{ Caractéristiques immuno-virologiques } \\
\hline \multicolumn{4}{|l|}{ CD4+Nadir } \\
\hline$<250$ & 122 & 68 & \multirow[t]{2}{*}{0,03} \\
\hline$\geq 250$ & 43 & 11 & \\
\hline \multicolumn{4}{|c|}{ CD4 à l'initiation de la $2^{\mathrm{e}}$ ligne } \\
\hline$<250$ & 83 & 37 & \multirow[t]{2}{*}{0,6} \\
\hline$\geq 250$ & 82 & 42 & \\
\hline \multicolumn{4}{|c|}{$\mathrm{CVp}$ à l'instauration de la $2^{\mathrm{e}}$ ligne } \\
\hline $1000-10000$ & $24(46,2)$ & $28(53,8)$ & \multirow[t]{3}{*}{0,003} \\
\hline $10001-100000$ & $47(78,3)$ & $13(21,7)$ & \\
\hline$>100000$ & $68(66,0)$ & $35(44,0)$ & \\
\hline
\end{tabular}

\section{Traitement ARV}

Délai de mise sous ARV de $1^{\mathrm{e}}$ ligne après la $1^{\mathrm{e}}$ consultation

$\leq 30$ jours

$>30$ jours

$123(82,0)$

$42(44,7)$

Combinaisons thérapeutiques de $1^{\mathrm{e}}$ ligne

AZT(D4T) + 3TC+EFV

TDF+3TC(FTC) + EFV

$\mathrm{AZT}(\mathrm{D} 4 \mathrm{~T})+3 \mathrm{TC}+\mathrm{LPVr}$

Durée de suivi du traitement ARV $1^{\mathrm{e}}$ ligne (ans)

Durée de suivi du traitement ARV $2^{\mathrm{e}}$ ligne (ans)

Combinaisons thérapeutiques de $2^{\mathrm{e}}$ ligne

$\mathrm{ABC}+3 \mathrm{TC}+\mathrm{LPVr}$

$\mathrm{ABC}+\mathrm{ddI}+\mathrm{LPVr}$

$\mathrm{TDF}+\mathrm{FTC}+\mathrm{DRV}+\mathrm{RTV}$

$\mathrm{TDF}+\mathrm{FTC}+\mathrm{LPVr}$
$96(56,5)$

$12(70,6)$

$4(100)$

$4,7(3,2-7,0)$

$2,8(2,6-3,0)$

$28(77,8)$

$48(64,9)$

$02(22,2)$

$87(69,6)$
$27(18,0)$

$52(55,3)$

$74(43,5)$

$5(29,4)$

$-$

$4,0(2,6-7,2)$

$2,2(1,1-3,5)$

0,3

0,2

$8(22,2)$

$26(35,1)$

$7(77,8)$

$38(30,4)$

Les données sont exprimées en moyenne \pm écart-type, (extrêmes) et en n (\%); CD4 en cellules/ $\mu 1$, copies/ml

Dans notre série, la CVp était supérieure à 10000 copies/ $\mathrm{ml}$ au début du traitement de deuxième ligne chez la plupart de nos patients. Ce fait est rapporté dans la littérature par certains auteurs $[5,13]$. Aussi, une étude publiée par Vitoria et al [16] note que plus la réplication virale initiale est importante, plus le risque de VPN est élevé. Une des hypothèses est que plus la charge virale initiale pré-thérapeutique est élevée, plus le réservoir de cellules infectées par le VIH est important, conduisant à un relargage de virus plus important en cas d'activation cellulaire, et à un risque plus important de persistance de la réplication virale à un bas niveau dans certains sanctuaires anatomiques $[1,15]$.

Dans notre structure, les stratégies de soutien accru à la prise des médicaments et la présence obligatoire du patient 


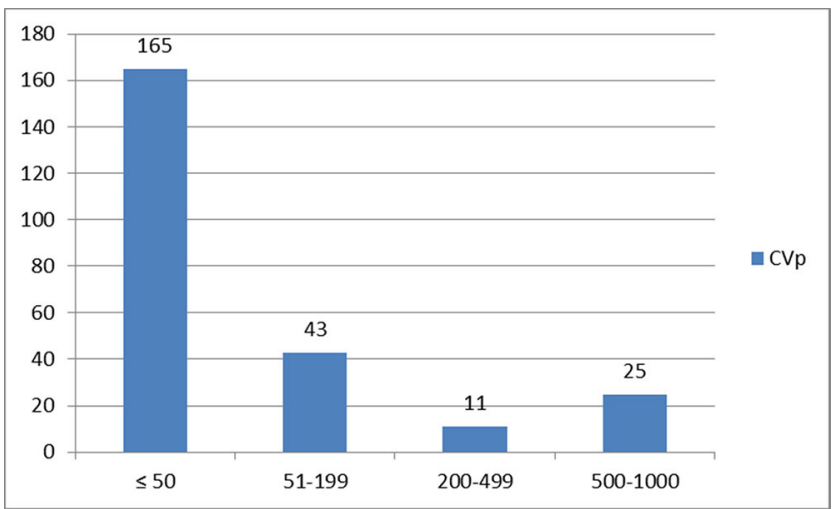

Fig.1 Répartition des patients selon la charge virale plasmatique en copies/ml / Distribution of patients by plasma viral load in copies/ml

dans le service une fois par mois, n'ont pas permis de contrôler cette virémie persistante. La réalisation d'un dosage pharmacologique des ARV pourrait être recommandée dans cette situation pour optimiser la réponse virologique. Lorsque la concentration plasmatique des ARV est basse, un défaut d'absorption ou une interaction médicamenteuse devraient être recherchés. L'étude de Widera et al [18] sur la VPN du VIH-1 corrobore cette hypothèse en notant que $25 \%$ de leurs patients avaient des concentrations plasmatiques des ARV basses probablement en raison d'une inobservance aux ARV. Le coût du dosage pharmacologique des ARV en limite la réalisation en Afrique sub-saharienne. La réalisation d'un test génotypique de résistance aux ARV serait également d'un grand apport en cas d'échec de la première ligne. Malheureusement ce test est tout aussi inaccessible pour la grande majorité de nos patients.

\section{Conclusion}

À Bobo-Dioulasso, la VPN chez les patients infectés par le VIH-1, représente un grave problème au regard de sa fréquence élevée (un tiers des patients). Les femmes jeunes, un Nadir de CD4 bas, une CVp très élevée avant l'initiation $\mathrm{du}$ traitement de deuxième ligne, un délai long de mise sous traitement $\mathrm{ARV}$ de deuxième ligne et un protocole contenant ténofovir + entricitabine + darunavir/ritonavir sont les principaux facteurs liés à cette problématique. La réalisation d'un test génotypique de résistance et une surveillance rapprochée tous les trois mois de la charge virale s'imposent pour ces patients, afin de mieux adapter les stratégies thérapeutiques.

Liens d'intérêts : Les auteurs déclarent ne pas avoir de liens d'intérêts.

\section{Bibliographie}

1. Delaugerre C, Gallien S, Flandre P, et al (2012) Impact of lowlevel viremia on HIV-1 drug-resistance evolution among antiretroviral treated-patients. PLoS One 7:e36673. doi: 10.1371/ journal.pone.0036673. Epub 2012 May 10.

2. Doyle T,Smith C, Vitiello P, et al (2012) Plasma HIV-1 RNA Detection Below 50 Copies $/ \mathrm{mL}$ and Risk of Virologic Rebound in Patients Receiving Highly Active Antiretroviral Therapy. Clin Infect Dis 54:724-32. doi: 10.1093/cid/cir936. Epub 2012 Jan 11

3. Feinberg J, Clotel B, Khuong M-A, et al (2013) Once-daily dolutegravir (DTG) is superior to darunavir/ritonavir (DRV/r) in antiretroviral-naïve adults: 48-week results from Flamingo (ING114915). In: Program and abstracts of the 53rd Interscience Conference on Antimicrobial Agents and Chemotherapy, Denver, Colorado, U.S. Abstract H-1464a

4. García-Gascó P, Maida I, Blanco F, et al (2008) Episodes of lowlevel viral rebound in HIV-infected patients on antiretroviral therapy: frequency, predictors and outcome. J Antimicrob Chemother 61:699-704. doi: 10.1093/jac/dkm516. Epub 2008 Jan 12.

5. Martin-Blondel G, Sauné K, Vu Hai V, et al (2012) Factors associated with a strictly undetectable viral load in HIV-1-infected patients. HIV Med 13: 568-73. doi: 10.1111/j.1468-1293. 2012.01012.x. Epub 2012 Mar 21

6. Navarro J, Caballero E, Curran A, et al (2016) Impact of lowlevel viraemia on virological failure in HIV-1-infected patients with stable antiretroviral treatment. Antivir Ther 21:345-52. doi: 10.3851/IMP3023. Epub 2016 Jan 12

7. Ouedraogo SM, Zoungrana J, Sondo A, et al (2014) Caractéristiques sociodémographiques, cliniques, biologiques, thérapeutiques et déterminants de la réponse immuno virologique chez les adultes infectés par le VIH, sous traitement antirétroviral à l'hôpital De jour de Bobo-Dioulasso (Burkina Faso). RAFMI, 12:1-44

8. Pujades-Rodríguez M, O'Brien D, Humblet P, Calmy A (2008) Second-line antiretroviral therapy in resource-limited settings: the experience of Médecins Sans Frontières. AIDS 22:1305-12. doi: 10.1097/QAD.0b013e3282fa75b9.

9. Présidence du Faso, Ministère de la Santé (2014) Normes et Protocoles de prise en charge médicale des PVVIH au Burkina Faso, 3e édition, $71 \mathrm{p}$

10. Présidence du Faso (2016) Rapport d'activité sur la riposte au sida au Burkina Faso. Rapport GARPR 2015 du Burkina Faso. 49 p

11. Riddler SA, Aga E, Bosch RJ, et al (2015) Continued Slow Decay of the Residual Plasma Viremia Level in HIV-1-Infected Adults Receiving Long-term Antiretroviral Therapy. J Infect Dis 213:556-60. doi: 10.1093/infdis/jiv433. Epub 2015 Sep 2

12. Rouzioux C, Avettand-Fenoel V, Autran B (2013) Réservoirs et réponses immunitaires au cours de l'histoire naturelle de l'infection à VIH-1. Virologie 17:157-68. doi:10.1684/vir.2013.0500

13. Vancoillie L, Demecheleer E, Callens S, et al (2014) Markers associated with persisting low-level viraemia under antiretroviral therapy in HIV-1 infection. J Antimicrob Chemother 69:1098103. doi: 10.1093/jac/dkt484. Epub 2013 Dec 12

14. Vandenhende M A, Ingle S, May M, et al (2015) Impact of low-level viremia on clinical and virological outcomes in treated HIV-1-infected patients. AIDS 293:373-83. doi: 10.1097/ QAD.0000000000000544

15. Vandenhende M A, Perrier A, Bonnet F, et al (2015) Risk of virological failure in HIV-1-infected patients experiencing low-level viraemia under active antiretroviral therapy (ANRS C03 cohort study). Antivir Ther 20:655-60. doi: 10.3851/IMP2949. Epub 2015 Mar 4. 
16. Vitoria M, Hill AM, Ford NP, and al (2016) Choice of antiretroviral drugs for continued treatment scale-up in a public health approach: what more do we need to know? J Int AIDS Soc 19:20504. doi: 10.7448/IAS.19.1.20504. eCollection 2016.

17. Walensky RP, Borre ED, Bekker LG, et al (2016) The Anticipated Clinical and Economic Effects of 90-90-90 in South Africa. Ann Intern Med165:325-33. doi: 10.7326/M16-0799. Epub 2016 May 31.
18. Widera M, Dirks M, Bleekmann B et al (2017) HIV-1 persistent viremia is frequently followed by episodes of low-level viremia. Med Microbiol Immunol 206:203-15. doi: 10.1007/s00430-0170494-1. Epub 2017 Feb 20

19. Yazdanpanah Y, Fagard C, Descamps D, et al (2009) High rate of virologic suppression with raltegravir plus etravirine and darunavir/ritonavir among treatment-experienced patients infected with multidrug-resistant HIV: results of the ANRS 139 TRIO trial. Clin Infect Dis 49:1441-9. doi: 10.1086/630210 